\title{
Criterios de éxito del emprendimiento femenino, desde la metodología de la cartografía social*
}

\author{
Success criteria for female entrepreneurship, from the methodology of social mapping. \\ Critérios de sucesso para o empreendedorismo feminino, a partir da metodologia de \\ mapeamento social
}

DOI: https://doi.org/10.21803/penamer.13.26.418

Luz Yarime Peña Ulloa

https://orcid.org/0000-0003-3628-6277

Yaneth Carolina Gamba Mora https://orcid.org/0000-0002-2029-1541

Luisa Fernanda Acosta Cortes https://orcid.org/0000-0003-3738-3159
¿Cómo citar este artículo?

Peña. L, Gamba, Y. \& Acosta, L. (2020). Criterios de éxito del emprendimiento femenino, desde la metodología de la cartografía social. Pensamiento Americano, 13(26), 17-32.

DOI: https://doi.org/10.21803/penamer.13.26.418

\section{Resumen}

Este artículo presenta los criterios de éxito de emprendimiento empresarial femenino en la comuna ocho de la ciudad de Villavicencio, a partir de los elementos metodológicos de la cartografía social, como: el territorio, los actores participantes, que se constituyen en la unidad de análisis y de trabajo, las relaciones con otros actores que intervienen en el ejercicio empresarial. Los resultados del mapeo participativo y de los elementos teóricos orientan la conformación de una línea base para el estudio sobre emprendimiento de género, a fin de visibilizar las experiencias exitosas de manera gráfica.

Palabras Clave: Emprendimiento, Género, Contabilidad, Cartografía Social, Criterios De Éxito.

\begin{abstract}
This article presents the criteria for the success of female entrepreneurial entrepreneurship in commune eight of the city of Villavicencio, based on the methodological elements of social cartography, such as: the territory, the participating actors, which constitute the unit of analysis and at work, relationships with other actors involved in the business exercise. The results of the participatory mapping and theoretical elements guide the formation of a baseline for the study on gender entrepreneurship, in order to make visible the successful experiences in a graphic way.
\end{abstract}

Keywords: Entrepreneurship, Gender, Accounting, Social Mapping, Success Criteria.

* El presente artículo es producto del proyecto de investigación titulado: Mapeo participativo de experiencias exitosas de mujeres, como sujeto activo de emprendimiento en la ciudad de Villavicencio, Código C01-F05-029-2017, Universidad de los Llanos. 


\section{Resumo}

Este artigo apresenta os critérios para o sucesso do empreendedorismo feminino na comuna oito da cidade de Villavicencio, com base nos elementos metodológicos da cartografia social, tais como: o território, os atores participantes, que constituem a unidade de análise e no trabalho, as relações com outros atores envolvidos nos negócios se exercitam. Os resultados do mapeamento participativo e dos elementos teóricos orientam a formação de uma linha de base para o estudo sobre empreendedorismo de gênero, a fim de tornar visíveis as experiências bem-sucedidas de forma gráfica.

Palavras-chave: Empreendedorismo, Gênero, Contabilidade, Mapeamento Social, Critérios De Sucesso.

\section{Perfiles}

Magister en Economía, docente Facultad de Ciencias Económicas, Universidad de los Llanos. E. Mail: Yarime.pena@unillanos.edu.co

18

Egresada programa Contaduría Pública, Universidad de los Llanos. E-mail: Yaneth. gamba@unillanos.edu.co

Egresada programa Contaduría Pública, Universidad de los Llanos. E-mail: luisa. acosta@unillanos.edu.co
Luz Yarime Peña Ulloa

Yaneth Carolina Gamba Mora

Luisa Fernanda Acosta Cortes 


\section{Introducción}

El empoderamiento de la mujer ha contribuido a su crecimiento y generar dinámicas de emprendimiento, que las instauran como sujetos participes del desarrollo social y económico en el territorio donde habitan. Algunos estudios como "La mujer en la gestión empresarial: Cobrando impulso en América Latina y el Caribe” de la Organización Mundial del Trabajo, OIT (2017), reconoce la participación de la mujer en los contextos laborales y en la dinámica empresarial; además en el documento se visibilizan los ejercicios de dirección e iniciativas empresariales. La Fundación Ewing Marion Kauffman, en su informe titulado "Sources of Economic Hope: Women's Entrepreneurship" (2014), se interesa en abordar el tema de las mujeres estadounidenses y el emprendimiento, no solo como una cuestión orientada al crecimiento económico; además el estudio, advierte sobre la preocupación de la participación de la población femenina educada, que no están involucradas en las dinámicas del crecimiento y la innovación empresarial.

Durante las últimas décadas en Colombia, se han implementado algunas estrategias con un enfoque de género, a fin de promover el emprendimiento femenino, para que sean partícipes de la economía nacional. De acuerdo con recientes investigaciones del Global Entrepreneurship Monitor, GEM (2017), la proporción de mujeres emprendedoras en Colombia creció un 68\% en el año 2016, a comparación del 2014.

Para fortalecer la dinámica emprendedora el Congreso colombiano decreta mediante la ley 1780 de 2016, propone como objetivo:

... impulsar la generación de empleo para los jóvenes entre 18 y 28 años, sentando las bases institucionales para el diseño y ejecución de políticas de empleo, emprendimiento y la creación de nuevas empresas jóvenes, junto con la promoción de mecanismos que impacten positivamente en la vinculación laboral con enfoque diferencial, para este grupo poblacional en Colombia (art. 1).
Algunos programas propuestos, se encuentran en los Planes Nacionales de Desarrollo, como el establecido para el periodo 2018-2022, que incluye "un pacto por la legalidad, el emprendimiento y un futuro con equidad para todos” (p.3), en las ocho líneas de política, contenidas en el programa denominado "Pacto de equidad para las mujeres", en la línea B: "Educación y empoderamiento económico para la eliminación de brechas de género en el mundo del trabajo”, estos programas se limitan a establecer estrategias para insertar a las mujeres en el medio laboral y a orientar apuestas para menguar las fisuras existentes entre géneros en el ámbito laboral y empresarial.

A nivel territorial en el Departamento del Meta, no se puede divisar este panorama con claridad. En el Plan de Desarrollo: “Tierra de Oportunidades. Inclusión-Reconciliación-Equidad”, 2016-2019, en la política denominada "Equidad y Género", incluye el programa de "Autonomía y Empoderamiento Económico de las Mujeres con enfoque étnico y diferencial”, este programa no propone estrategias y acciones para su desarrollo y el logro de la política a la que se refiere, se queda en la presentación de cinco productos para promover la obtención del empoderamiento económico de las mujeres en esta región. En cuanto a la capital del departamento, en la Política de "Equidad de Género y Empoderamiento de las Mujeres”, inserta en el Plan de Desarrollo Municipal 2016-2019: "Unidos podemos" (Concejo Municipal de Villavicencio, 2016), El documento se restringe a mostrar las dificultades de las mujeres en condición de pobreza, desplazamiento y violencia; además menciona "la necesidad de generar procesos para que se apropien de sus derechos sexuales y reproductivos y prevenir y erradicar la violencia basada en género" (p. 16); otros informes que presentan y publican información que tienen que ver con el emprendimiento femenino, lo muestran de manera general, clasificando por actividad económica, tamaño de las empresas, entre otros, pero no formulan programas serios para procurar el mejoramiento de las condiciones económicas de las mujeres, ni el impulso a sus negocios.

Con relación al panorama antes expuesto, la inves- 
tigación pretende a partir del soporte metodológico de la cartografía social, la construcción participativa de un mapeo, para develar las experiencias exitosas de mujeres como sujeto activo de emprendimiento en la comuna ocho de la ciudad de Villavicencio. El horizonte metodológico se soporta en un estudio de carácter Investigación Acción Participativa, IAP, que se materializa en dos tipos de mapas: uno topográfico y temático para la ubicación en un territorio de las empresarias con sus unidades empresariales y su actividad económica, otro mapa temático y de relaciones, en el que se representa los criterios de éxito y las interrelaciones con clientes, proveedores, instancias públicas y privadas.

El contenido del artículo se conforma de los siguientes apartados: primero de la fundamentación teórica y conceptual que soporta el objeto de estudio y el contexto del emprendimiento femenino como soporte del mapeo y la cartografía social, el método de investigación implementado, los resultados, las conclusiones y la discusión.

\section{Aproximación teórica}

Las teorías que fundamentan el objeto de estudio de la investigación son: género, emprendimiento y cartografía social.

La teoría de género se desglosa a partir del entendimiento del concepto de género, uno de los más complejos de sintetizar, debido a que se encuentra ligado a los imaginarios colectivos y representaciones sociales que establecen diferencias sexuales entre lo femenino y lo masculino, las relaciones de poder ubicadas en contextos y temporalidades, en otras palabras, no existe una definición única, si no que varía según la realidad social y cultural a la que se inscribe el concepto, que teóricos como Scott, Bourdieu y Foucault, contribuyeron en su construcción (Núñez, 2016).

Por tanto, el género como teoría está construida a partir de posturas heterogéneas y enfoques científicos, que a lo largo del tiempo se han fundamentado en distintas ideologías basadas en la epistemología feminista, que sustentan su concepción a partir de la victimización, con la mirada crítica hacia la opresión estructural, física, simbólica y la explotación económica de las mujeres, esta última descrita por Engels y Marx (Lagunas, Beltrán \& Ortega, 2016). Otros autores como Posada (2015), mencionan que el género como concepto, está arraigado al discurso y prácticas del poder en diversos escenarios, que se relaciona entre hombres y mujeres con la mirada objetiva de la comparación entre ambos, inclusive desde un sentido dialógico. (Martínez, 2018), afirma que hoy en día se le da una gran importancia al marketing, gracias al desarrollo de los mercados y evolución de clientes.

Para los propósitos de la investigación, no se tiene en cuenta el tema de género como la relación entre hombres y mujeres; tampoco desde la postura típica de la victimización, el interés es dar la oportunidad al entendimiento del empoderamiento femenino que las lleva a consolidar un ejercicio empresarial. El empoderamiento tiene sentido cuando se da significado y propósito al logro de la trasformación, es decir la superación de la subordinación de la mujer, frente a la representación de las estructuras de poder tradicionalmente establecidas Bethencourt y Carrillo (2014). Otra definición, pertinente para este estudio es el "empoderamiento económico de la mujer" (OXFAM Internacional, 2017), que cobra valor, en tanto:

Se da de manera real y efectiva cuando estas pueden ejercer su derecho a controlar y beneficiarse de los recursos, bienes e ingresos, así como a disponer de su propio tiempo, y cuando tienen la capacidad de gestionar los riesgos y de mejorar su situación económica y su bienestar. (p. 8)

Un concepto que se imbrica de manera ideal al empoderamiento femenino es el emprendimiento, como vía para lograr cambios significativos, orientados a transformar las relaciones existentes entre hombres y mujeres, el aporte de estas a su núcleo familiar y a la sociedad; principalmente desde el empoderamiento económico y social (Gallup, Inc., \& Organización Internacional del Trabajo, 2017). Teniendo en cuenta que el emprendimiento empre- 
sarial, es visto como motor de impulso para el crecimiento económico y la atención a las necesidades sociales como educación, empleo, salud, entre otros.

Quien ejerce la acción emprendedora, se tipifica como emprendedor, que para Lederman, Messina, Pienknagura y Rigolini, (2014), posee cualidades para "dirigir a otras personas, priorizar las tareas para aumentar la eficiencia productiva y darles a los recursos disponibles el mejor uso posible” (p. 1), concediendo de una u otra manera "poder" al emprendedor, que finalmente hace uso del empoderamiento.

Se entiende que la actividad emprendedora y el emprendedor en sí, encuentra su fundamento en un conocimiento construido con el devenir de su ejercicio y en su especialización, para tomar decisiones, en la utilización y administración de "recursos escasos", de manera oportuna, como lo refiere Casson (1982, citado en Terán \& Guerrero, 2020). Otras consideraciones conceptuales, ven al emprendedor como el sujeto que realiza "esfuerzos organizados" que los lleva a generar valor y crecimiento (Robbins, 2008, citado en Suárez, 2017) y como generador de nuevas empresas (Suárez, 2017).

Producto de la unión de las teorías de género y emprendimiento, es lo que se conoce como "emprendimiento de género" o "emprendimiento femenino"; autores como Kabeer (1999) y Sajardo, (2012) (Citados en Ribeiro, 2017); asocian el emprendimiento femenino al empoderamiento y lo sitúan en el campo del saber de la economía social, que no pretende quedarse en la cuantificación, sino que se preocupa en los efectos cualitativos de las emprendedoras.

Debido a que el emprendimiento tiene un rol decisivo en el desarrollo de las sociedades y las consecuencias que genera sobre el crecimiento económico de una región, en tal sentido el emprendimiento posee una relación directa entre el PIB de un país; es decir que se establece un bucle favorable para el crecimiento económico, que se materializa en la generación de auto empleo (Minniti, 2012).

\section{La cartografía social para la representación del emprendimiento}

La cartografía, no solamente se queda en la ubicación en un territorio, también alberga la posibilidad de representar mediante mapas físicos, las intencionalidades sociales y políticas que se requieren para dar sentido a los propósitos de la investigación.

La cartografía social (cs) en la que, inicialmente, a partir de las consideraciones espaciales del terreno se busca que las comunidades reconfiguren la territorialidad de los espacios habitados. Esta estrategia de intervención social y de investigación cobra cada vez más fuerza en diferentes campos de indagación. (Barragán, 2016, p. 250)

Como estrategia de investigación social, está ligada a un territorio, en sus límites físicos, entre sus interrelaciones y la comprensión que los sujetos tienen de este; además procura una construcción y reconfiguración colectiva de los elementos y las relaciones de los participantes en el mapeo. Algunos antecedentes de la cartografía social se refieren que se ha utilizado a manera de intervención e investigación en estudios de conflictos socio ambientales en comunidades afrodescendientes (Vélez, Retiva \& Varela, 2012); para la planeación territorial y gestión socio ambiental (Forero, 2015); para el análisis de los procesos del "activatorio de memoria y territorio”, (Mata, 2017, p. 52). En el área de conocimiento del emprendimiento, no se encontraron estudios con este enfoque.

Para los objetivos de la investigación, la cartografía social, permite reconocer la ubicación de las empresarias y sus unidades empresariales, establecer la interrelación de estas con los criterios de éxito empresarial propuestos, representados en símbolos iconográficos; además trascender en la construcción participativa del mapeo, que emerge de la información recabada en la aplicación de los instrumentos, atendiendo los preceptos de la investigación acción participativa, propuestos por Fals Borda como un método que busca producir conocimiento de forma colectiva y "reconoce y valora el saber del otro” (Moncayo, 2015, p. 336). 


\section{Criterios de éxito para el emprendimiento}

Para el estudio de la cuestión que interesa a la investigación, se escogen como criterios de éxito: la innovación, creación de empleo, factores financieros y de rentabilidad, formalización de la empresa, perdurabilidad empresarial y participación en redes de apoyo y contactos; estos criterios responden a la formación de las investigadoras en disciplinas de las ciencias económicas; que además se proponen como categorías de análisis en la interpretación del emprendimiento, que a continuación se explicitan:

Innovación. En el emprendimiento, una de la característica trascendental es la Innovación; autores como Hellriegel et al, (2005 citado en Garbanzo, 2016) la definen como "el proceso de crear y poner en práctica una idea nueva” (p. 82), Hage (1999 citado en Naranjo, Jiménez \& Sanz, 2012), precisan la innovación como desarrollar e implementar en el ámbito empresarial nuevas ideas o comportamientos. La innovación en el contexto empresarial se orienta a la generación de valor, que no se refiere solo a nuevos productos; también implica procesos o servicios. En tal sentido "El emprendimiento innovador es una actitud en las personas, una cultura y una capacidad en las empresas que en un entorno competitivo" (Vesga, 2009, citado en Acero, Ivanovic, Cano \& Luque, 2016, p. 20).

Creación de Empleo. Considerado como una contribución de las empresas a la sociedad, incluyendo el autoempleo; el fomento y consolidación del emprendimiento, hace más expedito que se materialice esta responsabilidad social. Las instancias supranacionales y estatales, propugnan por una cultura del emprendimiento, en respuesta a la creación de empleo, autoempleo y competitividad, que redunda en el mejoramiento del bienestar social (Valencia, 2012).

Factores financieros y de rentabilidad. Relacionado con los resultados obtenidos del buen manejo de los recursos financieros propios u obtenidos de manera externa, su buen uso se convierte en una medida de eficiencia económica, que propicia la creación y sostenimiento de su negocio, de esta manera realiza una transición de empleado a empresario (Salinas \& Osorio, 2012) y favorece el apalancamiento de su acción.

Formalización de la empresa. Entendido como un proceso que busca el cumplimiento de las normas dispuestas por los entes de inspección, vigilancia y control, para su aplicación, (Departamento Nacional de Planeación, 2017); además conocer los beneficios que trae la formalidad, para la perdurabilidad y rentabilidad de las empresas.

La perdurabilidad empresarial. Algunos teóricos como Peters y Waterman (1982); Pascale (1990) (Citados en Rivera, 2012), se refieren a la perdurabilidad orientada a las empresas que presentan factores de mejoramiento continuo, adaptación, mejoramiento en las tecnologías, procesos de comunicación, productividad, flexibilidad, relaciones con los clientes. Otros autores hacen referencia en la longevidad de las organizaciones, Collins \& Porras (1994); Hall (1997) (Citados en Rivera, 2012). Para los fines de la investigación se toma como perdurabilidad, la temporalidad de las unidades empresariales, fidelización y reconocimiento.

Participación en redes de apoyo y contactos. La oportunidad de contar con instancias externas del orden público y/o privado, se constituyen en una ayuda en procura de acceder a procesos de formación en temas propios de lo empresarial, fuentes de financiamiento, relaciones con sus proveedores y establecimiento de redes de apoyo y participación en organizaciones que promueven el emprendimiento (Cárdenas, Guzmán, Sánchez \& Vanegas, 2015).

\section{Contexto del emprendimiento femenino}

En Colombia la actividad empresarial femenina se ha fortalecido en los últimos años, de acuerdo con los resultados del informe presentado por Global Entrepreneurship Monitor, GEM (2017), las cifras de mujeres emprendedoras en el país aumentó en un 68\%, del $15 \%$ se incrementó al $25 \%$, y menciona que comparando esta cifra con las de otros países latinoamericanos y del Caribe, ha sido el de mayor crecimiento, producto de las estrategias que se han implementado desde el 
Gobierno Nacional, a partir de políticas para el fortalecimiento de género y participación femenina ("Emprendimiento femenino está en aumento”, 2019).

Para el caso del Departamento del Meta a partir del año 2008, se ha venido incluyendo en los planes de desarrollo la apuesta por el emprendimiento a partir de distintas perspectivas, que han evolucionado con el paso del tiempo, desde la creación de la cultura empresarial y el emprendimiento, la creación y el desarrollo de estos temas con la estrategia de "ruedas de empleo y emprendimiento con enfoque de género" (Universidad Nacional de Colombia, 2018, p. 263); de igual manera el Plan de Desarrollo Económico y Social del Departamento del Meta para el periodo 2016-2019, "El Meta: Tierra de Oportunidades" (Asamblea Departamental del Meta, 2016), propugna en uno de sus ejes trabajar por el "Desarrollo Humano Incluyente y Equitativo", este propone como metas fomentar y consolidar el emprendimiento de la mujer a partir de diversas apuestas productivas.

En coherencia con los planes a nivel departamental, el propósito de las apuestas de desarrollo para su capital Villavicencio, se queda en promover la participación de las mujeres, en el empoderamiento de estas para la erradicación de la violencia; pero no propone programas para la generación y fortalecimiento de ejercicios emprendedores.

En Villavicencio los sectores económicos que mayor participación tienen "son el comercio y los servicios, en conjunto agrupan al 86\%" (Cámara de Comercio de Villavicencio, 2014, p. 17). El comercio es el sector en el cual hay una mayor concentración de generación de empleo en un 53,1\%, seguido por hoteles y restaurantes con un $13,3 \%$ y en último lugar la industria manufacturera con un 9\%. Los sectores en los cuales la ciudad posee mayor capacidad de crecimiento son el sector productivo (agropecuario, agroindustria e industria) y el sector servicios (turismo y conexos), al igual con ellos puede generar mayor valor, (Cámara de Comercio de Villavicencio, 2014). Con relación a la información anterior, se puede observar que los datos emanados por las diferentes instancias acerca de la ac- tividad emprendedora, no la clasifica por género.

\section{Metodología}

La investigación se soporta en el método de Investigación Acción Participativa (IAP), de corte cualitativo, que involucra de manera activa a los grupos y organizaciones sociales. Entendiendo que esta perspectiva de investigación pretende el empoderamiento de los actores y visibiliza sus experiencias (Santos, 2003 citado en Hernández, 2013). La IAP, asume a los sujetos participantes en la investigación, como unidad de análisis y de trabajo; en tanto estos poseen unas condiciones que vale la pena interpretar a la luz de un análisis estructural, dado que evidencian características específicas; para la intencionalidad de esta investigación, se relacionan con el sujeto emprendedor, su empresa, contexto y territorio en el que se desarrollan sus acciones emprendedoras; por lo cual se dirigen los criterios de selección hacia las micro y pequeñas empresas, encabezadas por mujeres.

Lainformación se obtuvo con el diseñoy aplicación de una entrevista semiestructurada, que, de acuerdo con Díaz, Torruco, Martínez y Varela, (2013), es "una técnica de gran utilidad en la investigación cualitativa para recabar datos; se define como una conversación que se propone un fin determinado distinto al simple hecho de conversar" (p.163). Con este instrumento se recopilaron los datos aportados por la unidad de análisis y de trabajo, conformada por 19 mujeres que lideran sus respectivas empresas, estas reúnen el cumplimiento de los criterios de éxito y voluntariamente aceptaron hacer parte de esta investigación.

En la entrevista se solicitó información coherente con los criterios de éxito propuestos anteriormente, a través de la formulación de 44 preguntas, relacionadas con la representación legal, experiencia como empresaria, recursos tecnológicos, mejoras o cambios en los procesos, auto empleo, generación de empleo, fuente de financiamiento, factores de rentabilidad, formalización empresarial, sostenibilidad empresarial (temporalidad, fidelización, reconocimiento), contribución al desarrollo económico y vinculación y cohesión externa. 


\section{La cartografía y el territorio}

En tal sentido, se asume la comuna ocho de Villavicencio como marco geográfico, representado como territorio de esta investigación. El territorio en calidad de espacio físico delimitado, con características propias y la interacción de los actores que cohabitan en él (Gottmann, 1970 citado en González, 2011). En el contexto geográfico de estudio la interrelación de los actores conformados por las empresarias, sus unidades empresariales, los clientes, proveedores y la caracterización de la unidad de análisis y de trabajo que se articularon con la cartografía social, para representar los aspectos de éxito que las han consolidado y sostenido, estos se reflejan gráficamente en mapas, como elemento fundamental.

De esta forma, la cartografía social, se toma como una herramienta para generar conocimiento con un enfoque social - participativo. La finalidad de la cartografía se materializa en la construcción de mapas para ubicar geográficamente los objetos y sujetos e interrelacionar la información producto de la dinámica colectiva (Diez \& Rocha, 2016).

La elaboración de los mapas, que tiene relación con la proyección mental para ubicar las participantes localizadas al interior del territorio en el cual se desarrolló la investigación, incluye lugares físicos, con la intención de aproximarse a la realidad de los sujetos de estudio, identificar la unidad de análisis y de trabajo, sus interacciones, el lugar donde desarrollan las acciones emprendedoras y construir un lenguaje iconográfico para la comprensión de las categorías y subcategorías propuestas (Barragán, 2019).

\section{Resultados}

El análisis de la información cualitativa, requiere de la estructuración de categorías y subcategorías de análisis, la noción de categoría se refiere "son conceptos que hacen parte de la investigación, que surgen del marco teórico y que definen los conceptos que se van a utilizar en la investigación” (Monje, 2011, p. 92). En coherencia con el objeto de estudio las categorías de análisis se emplearon para estructurar los criterios de éxito y como conceptos que clarifican las categorías se proponen unas subcategorías, presentadas en la tabla 1, que nutren la información para la elaboración de la cartografía y representación en los mapas temáticos y de relaciones.

\section{Construcción del mapeo}

Con la información contenida en las entrevistas se representaron dos tipos de mapas: un mapa topográfico y temático y un mapa temático y de relaciones.

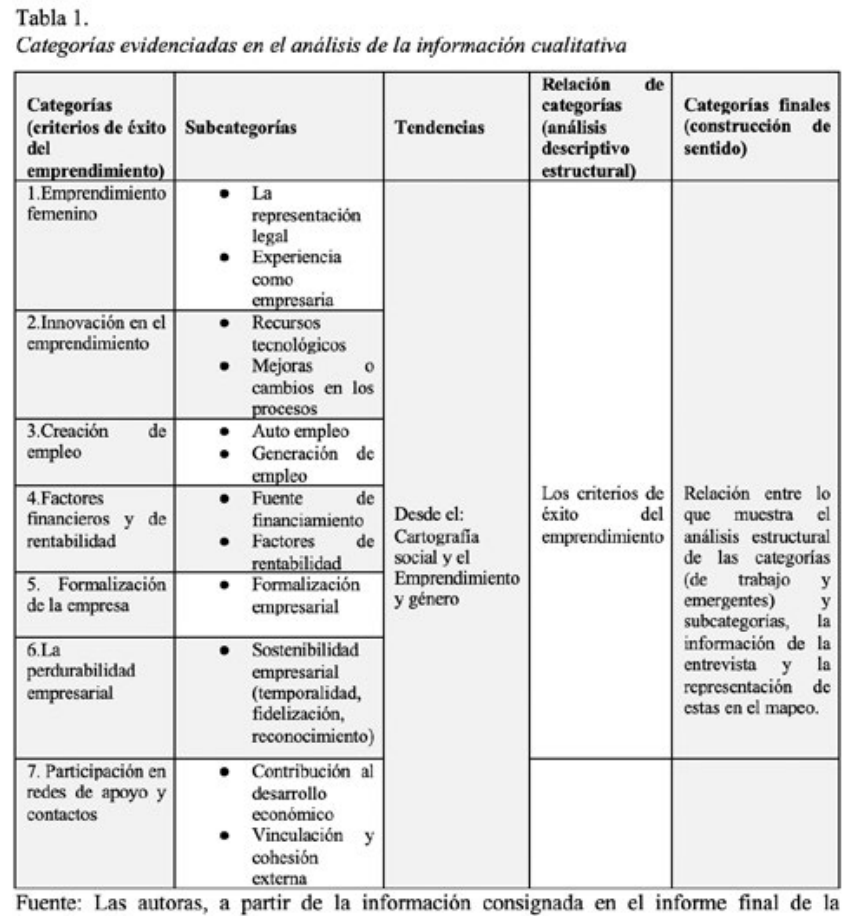
investigación.

El mapa topográfico y temático. Se concibe como el espacio geográfico del contexto de estudio y su representación, que corresponde a la delimitación de la superficie (Buzai, Lanzelotti, Paso \& Principi, 2018), en este caso parte de una zona urbana municipal, con la finalidad de ubicar sobre los mapas la representación iconográfica de la unidad de análisis y de trabajo, con su respectiva actividad económica, tal como se muestra en la tabla 2.

Para suministrar la ubicación y el registro de la información en los mapas, se procede a segmentar la co- 
muna ocho en dos secciones de la siguiente manera: el segmento 1, conformado por 14 barrios, se ubican en este espacio geográfico, 10 empresarias y sus unidades empresariales, como se representa en la figura 1 .

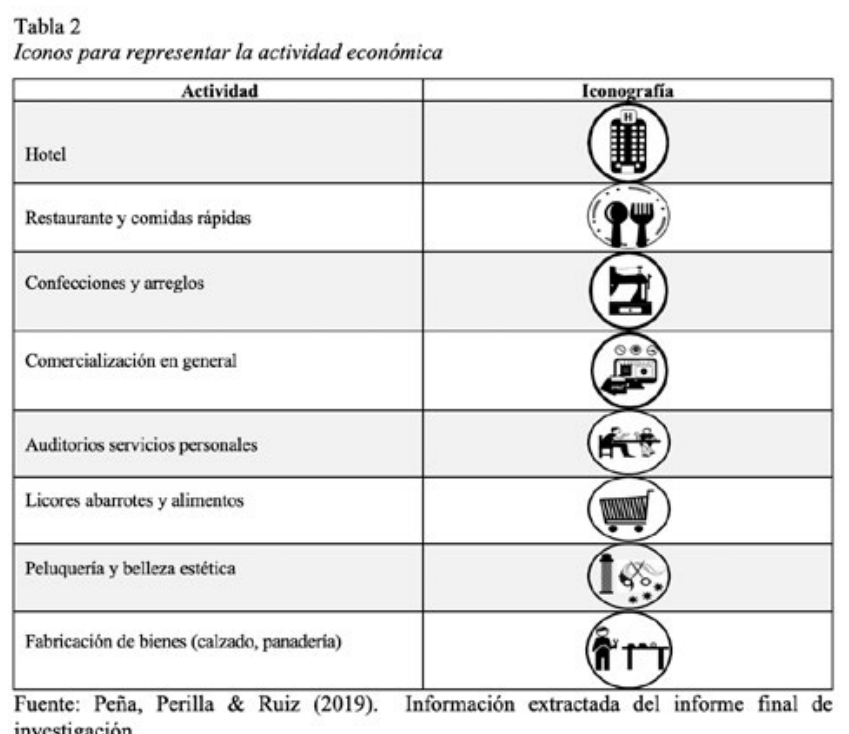
investigación.

La elaboración de los mapas permite el reconocimiento territorial por las emprendedoras, descubriendo una visión espacial de sus empresas, en el territorio geográfico de la comuna ocho. La figura 1, muestra la concentración de las unidades de negocio que se ubican en las áreas más pobladas, en las calles y avenidas principales de ingreso a la ciudad, con mayor flujo de personas y vehículos; esta condición favorece el desarrollo empresarial.

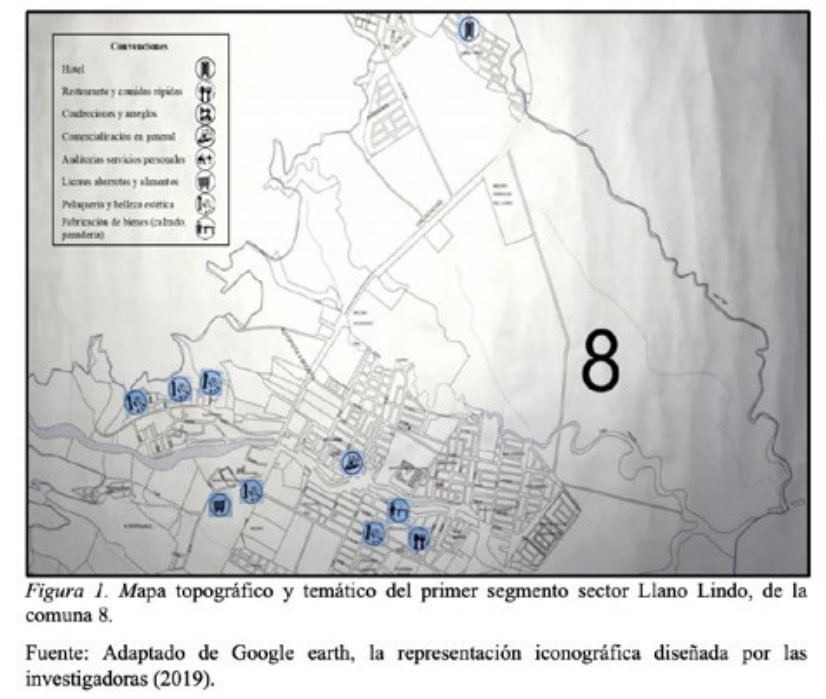

De igual forma, el mapa topográfico y temático identifica las actividades económicas que desarrollan las emprendedoras, como: hoteles, peluquerías, restaurantes y comercialización de víveres y abarrotes. Estas actividades empresariales se desarrollan en un entorno mixto entre la vida comercial y residencial, que es propiciado por las condiciones que se mencionaron anteriormente. De la unidad de trabajo participante, se encuentran en este espacio los siguientes negocios: un hotel, cinco salones de belleza, una panadería, un supermercado, una comercializadora de productos en general y una venta de comidas rápidas, actividades muy convenientes para ejercer las mujeres, por cuanto en el territorio se ubican cinco salones de belleza, situación que denota la inclinación del desempeño de ellas al sector servicios.

En este espacio geográfico se encuentran 9 emprendedoras, concentradas en dos zonas de este segmento, principalmente en los barrios Ciudad Porfía y Montecarlo, representados iconográficamente en el mapa en la parte inferior izquierda y superior derecha respectivamente, de igual forma, se denota que se sitúan de manera estratégica, donde existe una mayor densificación de viviendas y sectores poblados, que motiva el incremento de ventas e ingresos para las empresas.

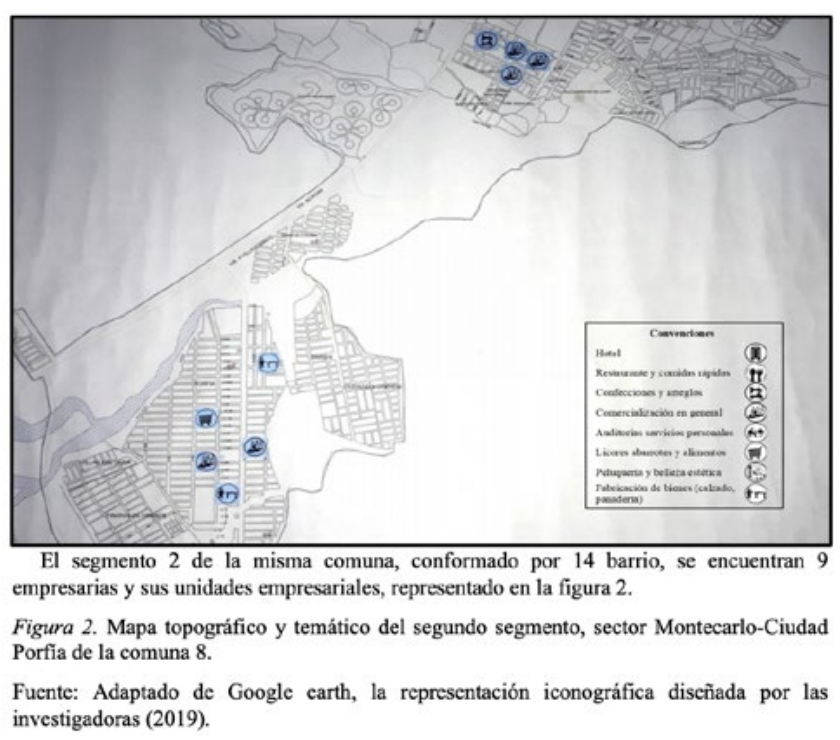

Igualmente, la figura 2 deja ver la diversidad y variedad de la naturaleza económica de los negocios es- 
tablecidos en esta zona, entre estos se destacan la comercialización de productos en general y los servicios. Otros aspectos que sobresalen a partir de la cartografía, es la Heterogeneidad de la estratificación socioeconómica de esta comuna, que corresponden a estratos 1,2 , 3 y 4 , situación que condiciona e incide en lo variopinto de los productos, y servicios ofrecidos y el tamaño de los negocios de las emprendedoras.

El mapa temático y de relaciones. Este contribuye a "ordenar la información del entorno social; además a registrar las redes relacionales que constituyen el territorio, construyendo así una imagen holística de la realidad" (Quiñonez,2011, p. 5), este tipo de mapa establece relaciones entre los siguientes elementos: la unidad de análisis y de trabajo, los criterios de éxito empresarial definidos en el marco teórico y conceptual, clasificados en categorías y subcategorías de análisis, que se presentan en la Tabla 1; además contribuye a graficar y hacer visible su interrelación con los actores y el aporte de estos a la actividad del emprendimiento empresarial femenino. Para este propósito, se diseñó una iconografía como se observa en la tabla 3 , que representa cada uno de los elementos mencionados.

Por el volumen de información a relacionar al interior de los mapas, las investigadoras tomaron la decisión de segmentar la comuna contexto de estudio y espacio geográfico, en tres mapas temáticos y de relaciones; estos se presentan en las figuras 3,4 y 5.

Tabla 3

Iconografia para representar las categorias y subcategorias de análisis

\begin{tabular}{|c|c|c|}
\hline Categorías de análisis & Subcategorías & \\
\hline \multirow{4}{*}{$\begin{array}{c}\text { Emprendimiento } \\
\text { femenino }\end{array}$} & La representación legal & \\
\cline { 2 - 3 } & Experiencia como empresaria & \\
\hline \multirow{2}{*}{$\begin{array}{l}\text { Innovación en el } \\
\text { emprendimiento }\end{array}$} & Recursos tecnológicos & \\
\cline { 2 - 3 } & Mejoras o cambios en los \\
procesos & \\
\hline \multirow{2}{*}{\begin{tabular}{c} 
Creación de empleo \\
\cline { 2 - 3 }
\end{tabular}} & Auto empleo & \\
\cline { 2 - 3 } & Generación de empleo & \\
\cline { 2 - 3 } & & \\
\hline
\end{tabular}

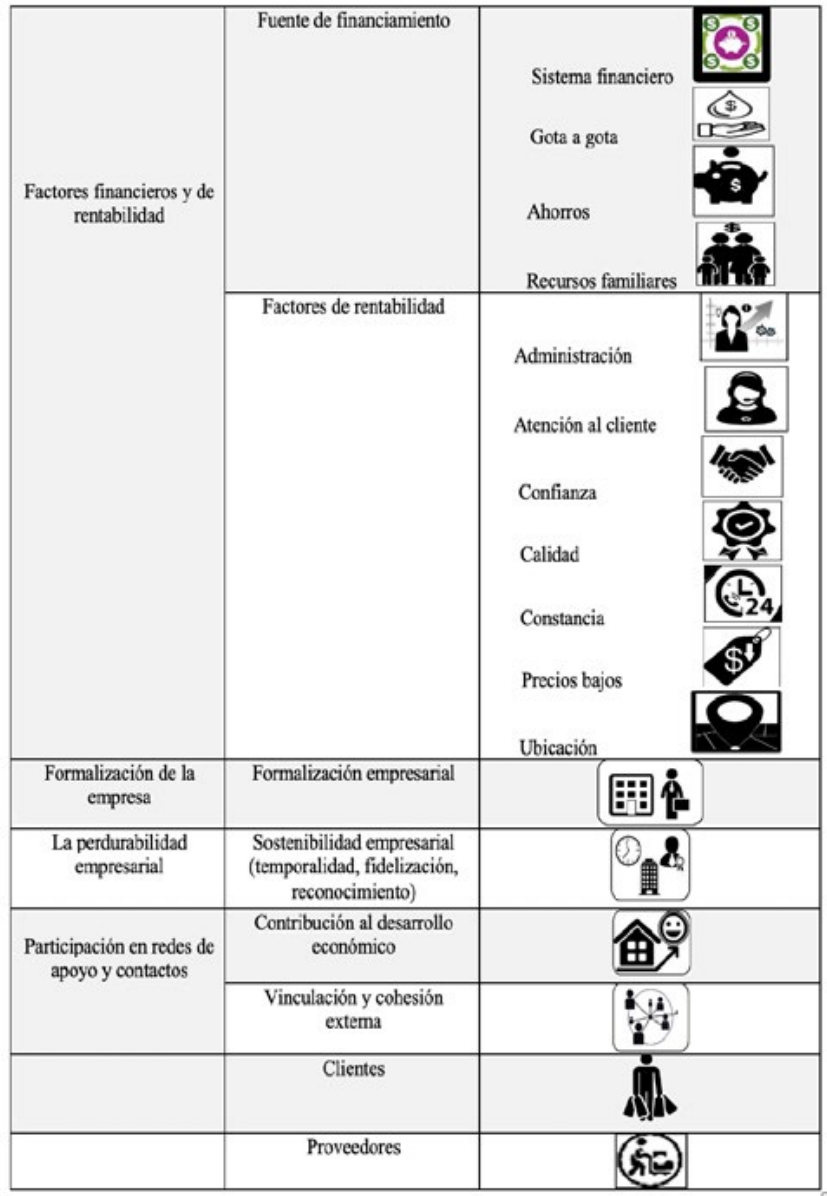

Fuente: Peña, Perilla \& Ruiz (2019). Información obtenida del informe final de la investigación.

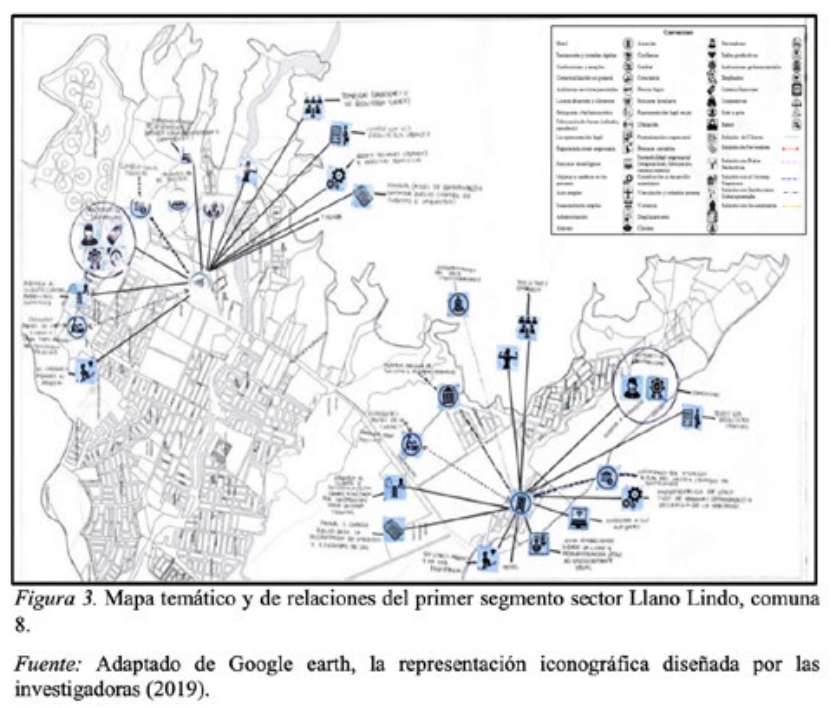

Para el caso del mapa 3 y de la zona en cuestión, aportaron información dos empresarias que tienen como actividad económica un hotel y un supermer- 
cado. El hotel, se encuentra en la margen inferior izquierda del mapa, sus representaciones iconográficas, se traducen a mostrar la siguiente información: de acuerdo con su propietaria, expone que los clientes en su mayoría provienen de diferentes zonas del país, especialmente de Bogotá y llegan con la finalidad de atender asuntos de negocios, de trabajo; en tal sentido las líneas de relación del hotel están punteadas y el icono de clientes esta por fuera del perímetro del mapa. El vínculo con los clientes, tiene una relación directa con la atención, calidad del servicio y posición espacial donde se encuentra el establecimiento; en cuanto a los criterios de empleo y autoempleo esta unidad de negocio genera empleo para sí misma y otras personas que viven en su comunidad, es visible gráficamente en la figura con una línea continua de relación, en la parte superior del icono del hotel.

Con relación a la otra unidad empresarial, el supermercado en este caso, identificado en la parte superior izquierda del mapa, es posible demostrar que los proveedores no están situados en la misma comuna, estos provienen del centro de la ciudad y de otras ciudades, situación que favorece el manejo por costos de mercancía y la calidad con respecto a la competencia, de acuerdo con lo expuesto por la empresaria: "mis proveedores se manejan también a nivel nacional en ciudades como Bogotá, Medellín, Cali y de la ciudad” (Comunicación personal, actor 4, febrero-2019).

La cartografía como perspectiva del territorio con enfoque social, representa en el mapa la relevancia de los factores de rentabilidad como criterios de éxito para las dos empresas; estos criterios están soportados en la atención al cliente, la calidad y los buenos precios, al interior del mapa se encuentran encerrados en círculos. La apuesta de las emprendedoras, por su empeño en la atención al cliente se recalca en los argumentos esgrimidos por estas "la atención al cliente es primordial en mi empresa, el manejo de las personas, la lealtad y amabilidad que muestro a mis clientes" (Comunicación personal, actor 2, febrero-2019).

También se simboliza de manera gráfica en el mapa 3 , la acción de poder y de auto superación como sub- categoría emergente, que se relaciona con la categoría de análisis de emprendimiento femenino, esta corresponde a una característica encontrada en algunas propietarias, quienes administran y representan legalmente su empresa. La condición de poder es un aspecto relacionado con el empoderamiento femenino, que le otorga autonomía para el logro de sus objetivos (Aguayo \& Hinrichs, 2015). Como lo menciona una de las entrevistadas, "yo soy la representante legal, la administradora y quien atiende el establecimiento" (Comunicación personal, actor 5, marzo-2019).

Desde otra arista, la investigación permitió mostrar el hallazgo de una subcategoría emergente, relacionada con el criterio de perdurabilidad, esta vez la experiencia de las mujeres en la actividad empresarial, con una tradición entre 10 y 20 años de permanencia en el

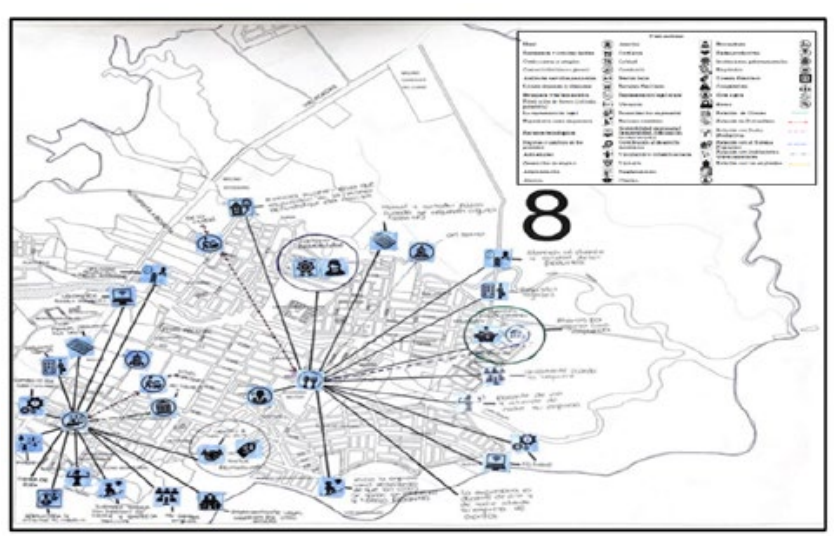

Figura 4. Mapa temático y de relaciones del segundo segmento sector la RochelaMontecarlo, de la comuna 8.

Fuente: Adaptado de Google earth, la representación iconográfica diseñada por las investigadoras (2019).

mercado. Su ejercicio empresarial se solventa a partir de una tradición familiar o por carencia de recursos económicos; este último motivado por la necesidad de ingresos para el sustento de su familia, referenciado por ellas mismas así: "tuve la oportunidad de ser empleada, pero prefiero ser independiente, pues me impulsa además mi condición de cabeza de familia" (Comunicación personal, actor 1, marzo-2019), testimonio que evidencia el deseo de autonomía económica.

En cuanto a las empresarias que se localizan en el 
sector del Barrio Ciudad Porfía (figura 5), se puede observar por medio de la interpretación de la iconografía inserta en el mapa, que los criterios de éxito preponderantes son los factores financieros y de perdurabilidad,

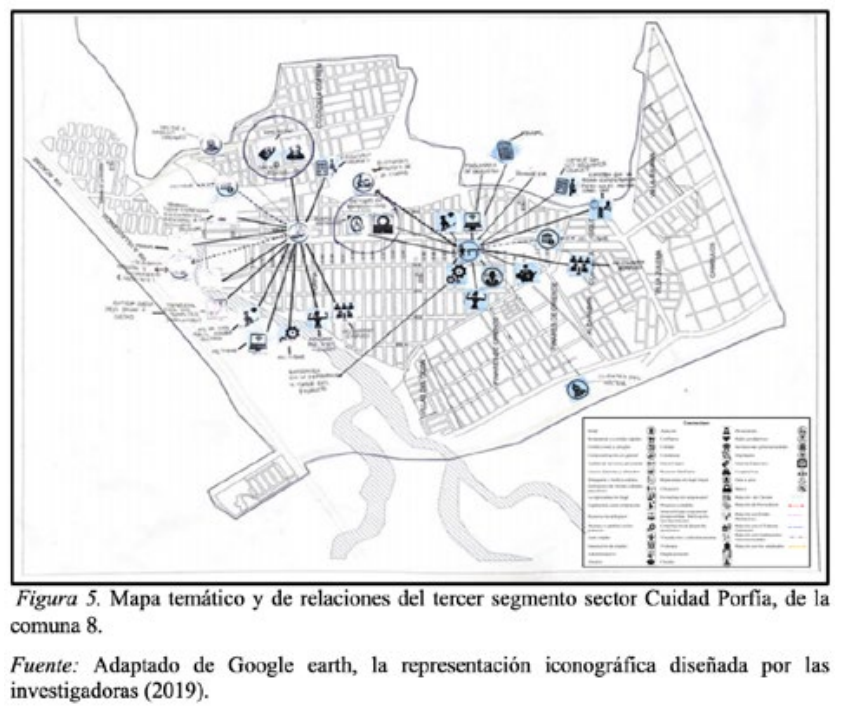

que radican en la atención al cliente y los precios bajos; sumado a los anteriores está la ubicación y la constancia de permanencia en el mercado. Otro criterio que las empresarias tienen en cuenta es la categoría de innovación, en especial la subcategorías de mejoras o cambios de procesos, en la prestación del servicio al cliente, recursos tecnológicos, estos últimos homologados por las empresarias como el manejo de las redes sociales, de acuerdo a la interpretación que le dan estas “ implementamos algunas nuevas estrategias como el uso de redes sociales como Facebook para la solicitud de los pedidos y se cuenta con domiciliario por WhatsApp” (Comunicación personal, actor 2, abril-2019).

Con relación a la categoría de generación de empleo, no es significativa, en cuanto a que la acción emprendedora se queda en la solución del empleo propio o autoempleo, este fenómeno se da por el tamaño de las empresas, que para el contexto de estudio es posible clasificarlas en microempresas según los criterios determinados por la ley 590 de 2000 y el decreto 957 de 2019. Los empleos que generan estas empresas son de 1 a 5 , procurando contratar a familiares y personas de su comunidad, sus empleados son contratados de manera temporal o a destajo, dependiendo de las tem- poradas de demanda de sus bienes o servicios.

Con relación a la categoría de participación en redes de apoyo y contactos, se ha dado un emprendimiento por interés propio y de carácter individual, en general las empresarias aducen que no han tenido colaboración de instancias gubernamentales y privadas que les oriente su trabajo y desarrollo empresarial, "no tengo ninguna red de apoyo, totalmente independiente" (Comunicación personal, actor 2, abril-2019), tan solo una menciona que, "hago parte de la Agencias de Turismos, y he participado en algunos eventos con la Alcaldía” (Comunicación personal, actor 3, abril-2019). Esta dinámica obedece a sus motivaciones, expectativas, atención del negocio y porque no han encontrado programas de orden gubernamental o de otras instancias, que les brinde garantías para la supervivencia y crecimiento de su ejercicio empresarial.

\section{Discusión}

Estudios de emprendimiento femenino, soportados en la metodología de cartografía social, carece de antecedentes relacionados con investigaciones, planes y estrategias gubernamentales, estos se enfocan en el emprendimiento a nivel general, a la presentación de cifras; para el caso de las mujeres, los programas de gobierno se orientan a atender las mujeres en condición de vulnerabilidad, coincidiendo, con autores como (Núñez, 2016), que aborda el concepto de género desde el discurso del poder en diversos escenarios.

La contribución de la cartografía social a la investigación, radica en la ubicación geográfica de las unidades empresariales y las relaciones de los sujetos emprendedores con otros actores y con el contexto circundante; además sirvió de escenario de representación y visibilización de los criterios de éxito para el emprendimiento femenino de las mujeres participantes. Se considera un estudio novedoso. "Todas las industrias están sujetas al efecto de las tendencias externas del tiempo”.(Salamanca, 2017)

Este tipo de investigación se ha trabajado en estudios de violencia, educación, de territorio y espacio; 
pero no se encuentran producción de conocimiento que aborde la cartografía y el emprendimiento.

El significado que se le da a los resultados del proyecto desmitifica la concepción de género a partir de la victimización de la mujer, que aborda (Lagunas, Beltrán \& Ortega, 2016), las investigadoras soportan esta argumentación, debido a que se encontró en las vivencias de las participantes un empoderamiento férreo hacia su ejercicio empresarial. Una subcategoría emergente tiene que ver con el crecimiento de sus unidades empresariales, a pesar que en el desarrollo de su emprendimiento no han tenido la colaboración de redes de apoyo e instancias que están obligadas a generar recursos y cooperación a esta actividad.

\section{Conclusiones y recomendaciones}

Los criterios de éxito empresarial esgrimidos por las empresarias participantes que conformaron la unidad de análisis y de trabajo son similares. Como factor común para lograr el emprendimiento, es la consecución de recursos financieros, las mujeres recurren a su propio pecunio, en especial los ahorros obtenidos en actividades diversas de ejercicios anteriores. Esta dinámica es recurrente debido al temor de contraer deudas y no poder cumplir con las obligaciones que estas demandan.

No existe una cultura de emprendimiento arraigada en las mujeres, debido a que los principales motivos para emprender de ellas, ha sido la necesidad de independencia, ligada a la obtención de ingresos para el sostenimiento propio y de sus familias, lo que se puede tomar como la apuesta por la búsqueda de la autonomía económica.

Se evidenció la no existencia de políticas gubernamentales y acciones de fortalecimiento orientados a este tipo de emprendimiento; no hay estructurado ningún programa de formación por instituciones e instancias del gobierno, que brinden solidez, respaldo y direccionamiento a las empresarias, la mayoría recurren a la experiencia adquirida en su acaecer propio del ejercicio.

\section{Referencias}

Acero, L., Ivanovic, C., Cano, J. \& Luque, S. (2016). Reflexiones sobre la política comercial y oportunidades de negocio para Colombia y Argentina. Recuperado de: https://bit.ly/3gPlk7Z

Aguayo, E. C. \& Hinrichs, J. S. (2015). Curadoras de semillas: entre empoderamiento y esencialismo estratégico. Revista Estudos Feministas, 23(2), 347-370. Recuperado de https:// dx.doi.org/10.1590/0104-026X2015v23n2p347

Asamblea Departamental del Meta. (2016). Ordenanza No.902 de 2016. Por medio de la cual se adopta el Plan de Desarrollo Económico y Social del Departamento del Meta para el periodo 2016-2019, "El Meta, Tierra de Oportunidades. Inclusión - Reconciliación - Equidad” y se dictan otras disposiciones. Recuperado de: http:/www.edesaesp.com.co/wp-content/ uploads/2016/07/No-902-MAYO-31-DE-2016-PLANDE-DESARROLLO-2016-2019-EL-META-TIERRA-DEOPORTUNIDADES.pdf

Barragán, A. N. (2019). Cartografía social: lenguaje creativo para la investigación cualitativa. Sociedad y Economía. (36), 139-159. Recuperado de https://sociedadyeconomia. univalle.edu.co/index.php/sociedad_y_economia/article/ view/7457/9909

Barragán, D. F. (2016). Cartografía social pedagógica: entre teoría y metodología. Revista Colombiana de Educación, (70), 247285. Recuperado de: http://www.scielo.org.co/pdf/rcde/ n70/n70al2.pdf

Bethencourt, L. \& Carrillo, M. (2014). Mujeres, empoderamiento y transformaciones socioeconómicas y políticas. Caso municipio Valdez, estado Sucre. Cuadernos del CENDES, 31 (85), 157-161. Recuperado de https://www.redalyc.org/ articulo.oa?id=403/40331800008

Buzai, G., Lanzelotti, S., Paso, L. F \& Principi, N. (2018). Cartografía analógica y digital para la delimitación regional y el análisis temático: aplicación a la cuenca del río Luján (Argentina). Revista de Geografía Norte Grande. (69), 99119. Recuperado de https://scielo.conicyt.cl/pdf/rgeong/ n69/0718-3402-rgeong-69-00099.pdf 
Cámara de Comercio de Villavicencio. (2014). Caracterización del tejido empresarial Villavicencio. Recuperado de: https://expeditiorepositorio.utadeo.edu.co/bitstream/handle/20.500.12010/1978/resumen\%20ejecutivo\%20villavicencio.pdf?sequence $=1 \&$ isallowed $=y$

Cárdenas, J., Guzmán, A., Sánchez, C. \& Vanegas, J. D. (2015). ¿Qué se crea al fomentar el emprendimiento? Los principales impactos de la formación en este campo. Universidad \& Empresa, 17(28), 173-190. Recuperado de: http://dx.doi. org/10.12804/rev.univ.empresa.28.2015.08

Concejo Municipal de Villavicencio (2016). Acuerdo 293 de 31 de mayo de 2016. Por el cual se adopta el Plan de Desarrollo Municipal 2016-2019, "Unidos Podemos", Recuperado de http://antigua.villavicencio.gov.co/index.php?option=com_docman\&task=cat_view\&gid=1773\&limit=100\&limitstart $=0 \&$ order $=$ hits $\&$ dir $=$ DESC $\&$ Itemid $=88$

Congreso de Colombia (02 de agosto de 2000). Por medio de la cual se modifica la Ley 590 de 2000 sobre promoción del desarrollo de la micro, pequeña y mediana empresa colombiana y se dictan otras disposiciones. [Ley 590 de 2000]. DO: 45.628. Recuperado de http://www.secretariasenado.gov. co/senado/basedoc/ley_0905_2004.html

Congreso de Colombia (2 mayo de 2016). Por medio de la cual se promueve el empleo y el emprendimiento juvenil, se generan medidas para superar barreras de acceso al mercado de trabajo y se dictan otras disposiciones. [Ley 1780 de 2016]. DO: 49861.

Departamento Nacional de Planeación (2017). Crecimiento $e$ informalidad en Colombia. Recuperado de: https://colaboracion.dnp.gov.co/cdt/prensa/crecimiento\%20e\%20informalidad\%20en\%20colombia.pdf

Díaz, L., Torruco, U., Martínez, M. \& Varela, M. (2013). La entrevista, recurso flexible y dinámico. Investigaciones en Educación Médica, 2(7):162-167 Recuperado de: https://www. redalyc.org/pdf/3497/349733228009.pdf

Diez, J. M. \& Rocha, E. (2016). Cartografía social aplicada a la intervención social en barrio Dunas, pelotas, Brasil. Revista Geográfica de América Central, 2 (57), 97-128. Recuperado de https://www.redalyc.org/pdf/4517/451748499005.pdf

Emprendimiento femenino está en aumento. (08 de marzo de 2019,). Revista Portafolio. Colombia. Recuperado de: https://www.portafolio.co/negocios/emprendimiento/emprendimiento-femenino-esta-en-aumento-519507

Forero, N. (2015). La cartografía social como herramienta de articulación entre la planeación territorial y la gestión socio ambiental. Caso cuenca río Salitre, Bogotá D.C. (trabajo de pregrado). Universidad de Ciencias Aplicadas y Ambientales, Bogotá, Colombia. Recuperado de: https://repository. udca.edu.co/bitstream/11158/413/1/LA\%20CARTOGRAF\%C3\%8DA\%20SOCIAL\%20COMO\%20HERRAMIENTA\%20DE\%20ARTICULACION\%20ENTRE\%20 LA\%20PLANECI\%C3\%93N\%20TERRITORIAL\%20 Y\%20LA\%20GESTI\%C3\%93.pdf

Fundación Ewing Marion Kauffman. (2014). Sources of Economic Hope: Women's Entrepreneurship. Recuperado de https:// www.kauffman.org/-/media/kauffman_org/research-reports-and-covers/2014/11/sources_of_economic_hope_ womens_entrepreneurship.pdf

Gallup, Inc. y la Organización Internacional del Trabajo (OIT). (2017). Hacia un futuro mejor para las mujeres en el trabajo: la opinión de las mujeres y de los hombres. Recuperado de: https://www.ilo.org/wcmsp5/groups/public/---dgreports/--dcomm/---publ/documents/publication/wcms_607487.pdf

Garbanzo, G. (2016). Desarrollo organizacional y los procesos de cambio en las instituciones educativas, un reto de la gestión de la educación. Revista Educacion, 40(1), 67-87. Recuperado de: https://www.scielo.sa.cr/pdf/edu/v40nl/22152644-edu-40-01-00067.pdf

Global Entrepreneurship Monitor (GEM). (2017). Estudio de la Actividad Empresarial, reporte para Colombia. Recuperado de: file://D:/USUARIO/Downloads/gemcolombia2017-2-71-ilovepdf-compressed-1548259767.pdf

González, A. R. (2011). Nuevas percepciones del territorio, espacio social y el tiempo. Un estudio desde los conceptos tradicionales (o clásicos) hasta su concepción en el siglo XXI. Recuperado de: http://webiigg.sociales.uba.ar/iigg/jove- 
nes_investigadores/6jornadasjovenes/eje\%206\%20pdf/ eje\%206_gonzalezale.pdf

Hernández, R. (2013). Diagnóstico participativo con cartografía social. Innovaciones en metodología investigación-acción participativa (IAP). Revista Andaluza de Ciencias Sociales, (12). 53-65. Recuperado de: http://institucional.us.es/revistas/anduli/12/art_3.pdf

Lagunas, M., Beltrán, L. F. \& Ortega, A. (2016). Desarrollo, feminismo y género: cinco teorías y una canción desesperada desde el Sur. Revista Estudios del Desarrollo Social: Cuba y América Latina. 4(2). 62-75. Recuperado de http://scielo. sld.cu/pdf/reds/v4n2/reds06216.pdf

Lederman, D., Messina, J., Pienknagura, S. \& Rigolini, J. (2014). El emprendimiento en América Latina: muchas empresas y poca innovación. Resumen. Banco Mundial. Recuperado de: https://www.worldbank.org/content/dam/worldbank/document/lac/emprendimientoamericalatina_resumen.pdf

Martínez Juvené, J., Fontalvo Cerpa, W., \& Cantillo Guerrero, E. (2018). Uso de los atributos de un producto para la seducción de un cliente durante el proceso de compra. Dictamen Libre, 2(23), 91-106.

Mata, C. (2017). Cartografía Social como herramienta para el cumplimiento del Derecho a la Ciudad: la experiencia de “Ciudad Comuna” en dos barrios de la comuna 8 de Medellín, Colombia. (Trabajo de Maestría). Universidad Politécnica de Valencia, España. Recuperado de: https://riunet.upv.es/ bitstream/handle/10251/85351/memoria_44803900.pdf?sequence $=1$

Ministerio de Comercio, Industria y Turismo. (5 de junio de 2019). "Por el cual se adiciona el capítulo 13 al Título 1 de la Parte 2 del Libro 2 del Decreto 1074 de 2015, Decreto Único del Sector Comercio, Industria y Turismo y se reglamenta el artículo $2^{\circ}$ de la Ley 590 de 2000, modificado por el artículo 43 de la Ley 1450 de 2011."[Decreto 957 de 2019]. Recuperado de https://www.mincit.gov.co/getattachment/555adb9d-8a48-45f3-a2a5-lee9b35b2d09/Decreto-957-Porel-cual-se-adiciona-el-capitulo-13.aspx

Minniti, M. (2012). El emprendimiento y el crecimiento econó- mico de las naciones. Economía industrial, (383), 23-30. Recuperado de: https://www.mincotur.gob.es/Publicaciones/ Publicacionesperiodicas/EconomiaIndustrial/RevistaEconomiaIndustrial/383/Mar\%C3\%ADa\%20Minnit.pdf

Moncayo, V. M. (2015). Orlando Fals Borda, Una Sociología Santi pensante para América Latina. Buenos Aires, Argentina: Clacso, Recuperado de: http://biblioteca.clacso.edu.ar/ clacso/se/20151027053622/AntologiaFalsBorda.pdf

Monje, C. A. (2011). Metodología de la Investigación Cuantitativa y Cualitativa, Guía didáctica. Universidad Surcolombiana. Recuperado de https://www.uv.mx/rmipe/files/2017/02/ Guia-didactica-metodologia-de-la-investigacion.pdf

Naranjo, J., Jiménez, D. \& Sanz, R. (2012). ¿Es la cultura organizativa un determinante de la innovación en la empresa? Cuadernos de Economía y Dirección de la Empresa, 15, (2), 3-72. Recuperado de: https://reader.elsevier.com/reader/sd/pii/ S1138575811001113?token=9E002DD76CF88E61A4AE987326BA6FDF3280EB60A1B9DB27A368CE6C0D78606F2B39F5AAD06BE704FDAD4937ED9E9A9C

Núñez, G. (2016). Los estudios de género de los hombres y las masculinidades: ¿qué son y qué estudian? Culturales, 4(1), 9-31. Recuperado de http://www.scielo.org.mx/scielo.php?scrip$\mathrm{t}=$ sci_arttext\&pid=S1870-11912016000100009\&lng=es\&tlng=es.

Organización Internacional del Trabajo, OIT. (2017). La mujer en la gestión empresarial: Cobrando impulso en América Latina y el Caribe. Recuperado de: https:/www.ilo.org/ public/spanish/dialogue/actemp/downloads/events/2017/ lima_conf/wibm_fullreport_2017_sp.pdf

OXFAM Internacional. (2017). Marco conceptual de Oxfam sobre el empoderamiento económico de las mujeres (WEE). Recuperado de DOI: 10.21201/2017.9682 Ihttps://oxfamilibrary.openrepository.com/bitstream/handle/10546/620269/gt-framework-womens-economic-empowerment-180118-es.pdf?sequence=9\&isAllowed $=\mathrm{y}$

Peña, L. Y., Perilla, L \& Ruiz, M. C. (2019). Mapeo participativo de experiencias exitosas de mujeres como sujetos activos de emprendimiento en la ciudad de Villavicencio. (Código 
C01-F05-029-2017). Universidad de los Llanos.

Posada, L. (2015). El "género" Foucault y algunas tensiones feministas. Estudios de Filosofía, (52), 29-43. Recuperado de http://www.scielo.org.co/pdf/ef/n52/n52a03.pdf

Quiñonez, M. (2011). Una experiencia de cartografía social en la zona de bajamar -isla de cascajal buenaventura. Entramado. 7 (2), 156-17l. Recuperado de: http://www.scielo.org.co/ pdf/entra/v7n2/v7n2al0.pdf

Ribeiro, D. (2017). Empoderamiento femenino e inserción por la economía: análisis comparativo del modelo de las cooperativas andaluzas y de la economía solidaria en Brasil. (Tesis Doctoral). Universidad de Valencia, España. Recuperado de: http://base.socioeco.org/docs/tese_pdf_21-042017.pdf

Rivera, H. (2012). Perdurabilidad empresarial: concepto, estudios, hallazgos. Cuadernos de administración. 28(47). 105115. Recuperado de: http://www.scielo.org.co/pdf/cuadm/ v28n47/v28n47a09.pdf

Rodríguez, D. (2016). Emprendimiento sostenible, significado y dimensiones. Revista Katharsis, (21), 419-448. Recuperado de: http://revistas.iue.edu.co/index.php/katharsis

Salamanca Ospina, E. J., Uribe, C. A., \& Mendoza Vega, L. M. (2017). ¿Cómo desarrollar un nuevo mercado donde la competencia no tiene ninguna?. Dictamen Libre, (20), 107-120

Salinas, F. \& Osorio, L. (2012). Emprendimiento y Economía Social, oportunidades y efectos en una sociedad en transformación. CIRIEC-España, Revista de Economía Pública, Social y Cooperativa, (75), 129-151. Recuperado de: http:// base.socioeco.org/docs/_pdf_174_17425798008.pdf

Suárez, F. (2017). Reflexiones para la interpretación del emprendimiento como elemento potenciador de la calidad de vida en poblaciones de pobreza extrema. Revista Espacios. 38(53), 29-39. Recuperado de: https://www.revistaespacios.com/al7v38n53/al7v38n53p29.pdf

Terán, E. \& Guerrero, A. (2020). Teorías de emprendimiento: revisión crítica de la literatura y sugerencias para futuras investigaciones. Revista Espacios. 41(7), 7-23. Recupe- rado de: https://www.revistaespacios.com/a20v4ln07/ a20v4ln07p07.pdf

Universidad Nacional de Colombia. (2018). Ecosistemas regionales del emprendimiento en Colombia. Recuperado de: https://innpulsacolombia.com/sites/default/files/2.2_mapeo_e_infografia.pdf

Valencia, G. D. (2012). Autoempleo y emprendimiento. Una hipótesis de trabajo para explicar una de las estrategias adoptadas por los gobiernos para hacer frente al progreso del mercado. Semestre Económico, 15(32), 103-128. Recuperado de http://www.scielo.org.co/pdf/seec/vl5n32/v15n32a5.pdf

Vélez, I., Retiva, S. \& Varela, D. (2012). Cartografía social como metodología participativa y colaborativa de investigación en el territorio afrodescendiente de la cuenca alta del río Cauca. Cuadernos de Geografía - Revista Colombiana de Geografía, 21, (2). 59-73. Recuperado de: http://www.redalyc.org/ pdf/2818/281823592005.pdf
2020, Vol. 13(26) 17-32 (CThe Author(s) 2020

Reprints and permission: www.americana.edu.co http://publicaciones.americana.edu.co/index.php/pensamientoamericano/index 